\title{
The lin-3/let-23 pathway mediates inductive signalling during male spicule development in Caenorhabditis elegans
}

\author{
Helen M. Chamberlin* and Paul W. Sternberg \\ Howard Hughes Medical Institute, Division of Biology, California Institute of Technology, Pasadena, CA 91125, USA \\ *Present address: Institute of Molecular Biology and Biochemistry, Simon Fraser University, Burnaby, BC, V5A 1S6 Canada
}

\section{SUMMARY}

During Caenorhabditis elegans male spicule development, four pairs of precursor cells respond to multiple positional cues and establish a pattern of fates that correlates with relative anterior-posterior cell position. One of the extracellular cues is provided by the $F$ and $U$ cells, which promote anterior fates. We show that the genes in the lin3/let-23 signalling pathway required for hermaphrodite vulval induction also mediate this F/U signal. Reductionof-function mutations in lin-3, let-23, sem-5, let-60 or lin-45 disrupt the fate of anterior cells. Likewise, activation of the pathway with ubiquitously produced signal results in posterior cells inappropriately adopting the anterior fates even in the absence of $F$ and $U$. We have further used this genetic pathway to begin to understand how multiple posi- tional cues are integrated to specify cell fate. We demonstrate that lin-15 acts in spicule development as it does in vulval induction, as a negative regulator of let-23 receptor activity. A second extracellular cue, from Y.p, also acts antagonistically to the lin-3/let-23 pathway. However, this signal is apparently integrated into the lin-3/let-23 pathway at some step after lin-45 raf and is thus functionally distinct from lin-15. We have also investigated the role of lin-12 in forming the anterior/posterior pattern of fates. A lin-12 gain-of-function defect is masked by redundant positional information from $F$ and $U$.

Key words: cell lineage, cell interaction, cell fate specification, pattern formation

\section{INTRODUCTION}

Cell interactions play a central role in the development of many organisms. As the specific proteins and cellular processes involved in classic vertebrate inductions become identified, the underlying complexities of multiple signals that act in parallel to specify fates are striking (reviewed in Kimelman et al., 1992; Davidson, 1993). A key issue in understanding how fate is specified by multiple signals is understanding how the information content of such signals is integrated in the responding cells to produce a unified outcome. In $C$. elegans, the essentially invariant and precisely described cell lineage provides a reproducible background to study cell interactions. The set of characterized interactions required during the development of the male spicules indicate that they may serve as a model that combines complex signal integration with the precision of single cell analysis (Chamberlin and Sternberg, 1993).

During $C$. elegans male postembryonic development, several precursor cells divide in a sex-specific manner to produce the cells of copulatory structures in the tail. One such cell, $\mathrm{B}$, is the precursor of all of the cells of the male spicules (Fig. 1A). During male development, the anterior daughter of B (B.a) divides to produce eight progeny (Fig. 2). These eight progeny make up four pairs: ventral (aa), dorsal (pp) and two identical lateral pairs (ap/pa). For each pair, there is an anterior and a posterior fate that differ in the subsequent cell lineage produced by each cell, as well as the differentiated fates of the progeny produced by that lineage (Sulston and Horvitz, 1977; Sulston et al., 1980). The choice of fate within each pair is responsive to extracellular cues provided by neighboring cells. Cell ablation experiments suggest that the other male-specific blast cells, or their progeny, provide distinct positional cues and that the eight B.a greatgrandprogeny may also interact (Chamberlin and Sternberg, 1993) (Fig. 3). For example, ablation of two other male-specific blast cells, called $F$ and $U$, disrupts the fates of positionally anterior cells. In some cases they produce the cell lineage normally associated with their posterior neighbors. In contrast, ablation of the blast cell Y.p disrupts the fate of some positionally posterior cells.

To identify the genes that mediate the cell interactions required for proper development of the $\mathrm{B}$ cell, we have begun to characterize the role of genes required for other cell interactions in C. elegans. In this study, we have focused on a set of genes that play a role in vulval development. C. elegans hermaphrodite vulval development requires a signal from the anchor cell (AC) in the gonad that acts on three of six epidermal blast cells termed vulval precursor cells (VPCs) (Kimble, 1981). In normal development, the three ACproximal VPCs produce vulval tissue, whereas the three distal VPCs produce nonspecific epidermis. The AC signal (an epidermal growth factor (EGF)-like protein encoded by the gene lin-3 (Hill and Sternberg, 1992)) is both necessary and sufficient to promote the VPCs to initiate vulval development. Genes that are necessary for the response to lin-3 include let- 

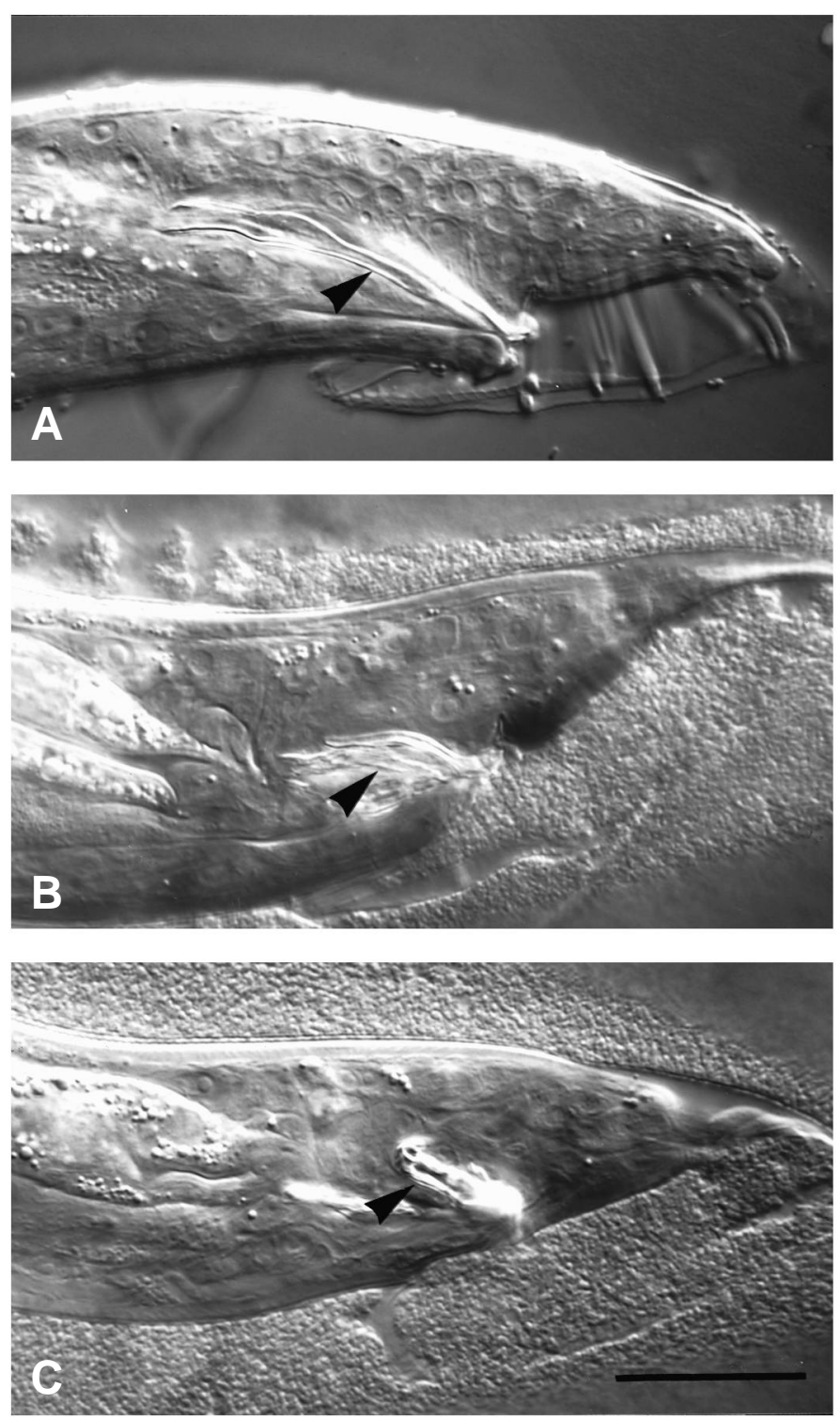

Fig. 1. Comparison of the adult male spicules in wild-type (A), lin3(sy53)/lin-3(n1058) (B) and syEx21(hsp::lin-3) (C) animals. Arrow points to the left spicule. Nomarski photomicrographs, anterior left, ventral down. (A) In wild-type animals, the spicules are long and straight. (B,C) In animals with lin-3 activity reduced or with lin-3 activity increased, the spicules are short and crumpled. However, the B lineage defects responsible for the morphological defect are opposite from each other (see text). Scale, $20 \mu \mathrm{m}$.

23 (receptor) (Aroian et al., 1990), sem-5 (adaptor) (Clark et al., 1992), let-60 (ras) (Han and Sternberg, 1990) and lin-45 (raf) (Han et al., 1993). Reduction-of-function mutations in any of these genes result in a Vulvaless (Vul) phenotype where all six VPCs may produce non-vulval epidermis at the expense of vulval tissue. Gain-of-function mutations in let-60 (Beitel et al., 1990) and over-production of LIN-3 (Hill and Sternberg, 1992) result in a Multivulva (Muv) phenotype in which all six VPCs may produce vulval tissue.

A second potential cell interaction is represented by the gene lin-15. Loss-of-function mutations in lin-15 result in a Muv phenotype. In vulval development, genetic analysis suggests that lin-15 acts in parallel to lin-3, as a negative regulator of let-23 (Ferguson et al., 1987; Huang et al., 1994). Genetically, lin-15 represents two independently mutable functions both of which must be mutant to produce an observable phenotype (Ferguson and Horvitz, 1989). Mosaic analysis indicates that lin-15 activity in the VPCs or in the AC is not sufficient for normal vulval development, and it is proposed that lin-15 may function in the surrounding epidermis (hyp7) (Herman and Hedgecock, 1990). The cloning of lin-15 revealed a complex locus with two transcriptional units that each have the potential to encode a novel protein (Huang et al., 1994; Clark et al., 1994).

In normal vulval development, the induced VPCs adopt one of two vulval fates. The cell most proximal to the AC adopts a $1^{\circ}$ fate, whereas the two more distal adopt a $2^{\circ}$ vulval fate. A lateral interaction between VPCs ensures that two adjacent cells do not adopt the $1^{\circ}$ fate (Sternberg, 1988). The gene lin12 mediates this lateral interaction and acts in several cell pairs as a genetic 'switch' between two fates (Greenwald et al., 1983). lin-12 acts in a cell-autonomous manner, likely on the receiving end of a cell interaction (Seydoux and Greenwald, 1989). The cloning of lin-12 revealed a transcript with the potential to encode a transmembrane protein with thirteen EGF-like repeats in the presumed extracellular domain, six copies of a cdc10/SWI6 motif in the presumed intracellular domain and overall similarity to the Drosophila gene Notch (Yochem et al., 1988).

In hermaphrodite vulval development, the three cell interactions represented genetically by the lin-3/let-23 pathway, lin15 and lin-12 act coordinately to produce the normal pattern of cell fates (reviewed by Sternberg, 1993). In this paper, we describe experiments that investigate the role of these genes in specifying fate in the male B lineage. We also investigate the relationship between the cell interactions identified by cell ablation experiments and the genetic pathways. Although the specifics differ between the two developmental processes, the function of these genes in the B lineage parallels that of vulval development: the genes of the lin-3/let-23 pathway mediate a positional signal from the $\mathrm{F}$ and $\mathrm{U}$ cells, lin-15 likely acts as a negative regulator of let-23 (receptor) activity and lin-12 mediates a lateral interaction. Furthermore, in the B lineage, ablation of the Y.p cell defines an additional cell interaction distinct from these three genetic pathways.

\section{MATERIALS AND METHODS}

\section{Strains}

Nematode strains were cultured according to standard techniques (Brenner, 1974; Sulston and Hodgkin, 1988). Loss-of-function mutations in lin-3, let-23, sem-5, let-60 and lin-45 are known or believed to be lethal, with the death phase prior to the time of spicule development. However, we believe the genotypes in Table 1B represent a reduction of normal gene function based on two criteria. First, for male tail development each allele is recessive to a wild-type copy of the gene (except for let-60(sy95dn) and let-60(sylo0dn); data not shown). Second, more stringent genetic tests indicate that the alleles represent a reduction of normal function of the gene for vulval development (Aroian and Sternberg, 1991; Clark et al., 1992; Ferguson and Horvitz, 1985; Han et al., 1990, 1993). For lin-3, let23 and let-60, we have tested several alleles or allelic combinations in order to verify that the observed phenotypes reflect common pleiotropies of the locus rather than unrelated second mutations or 

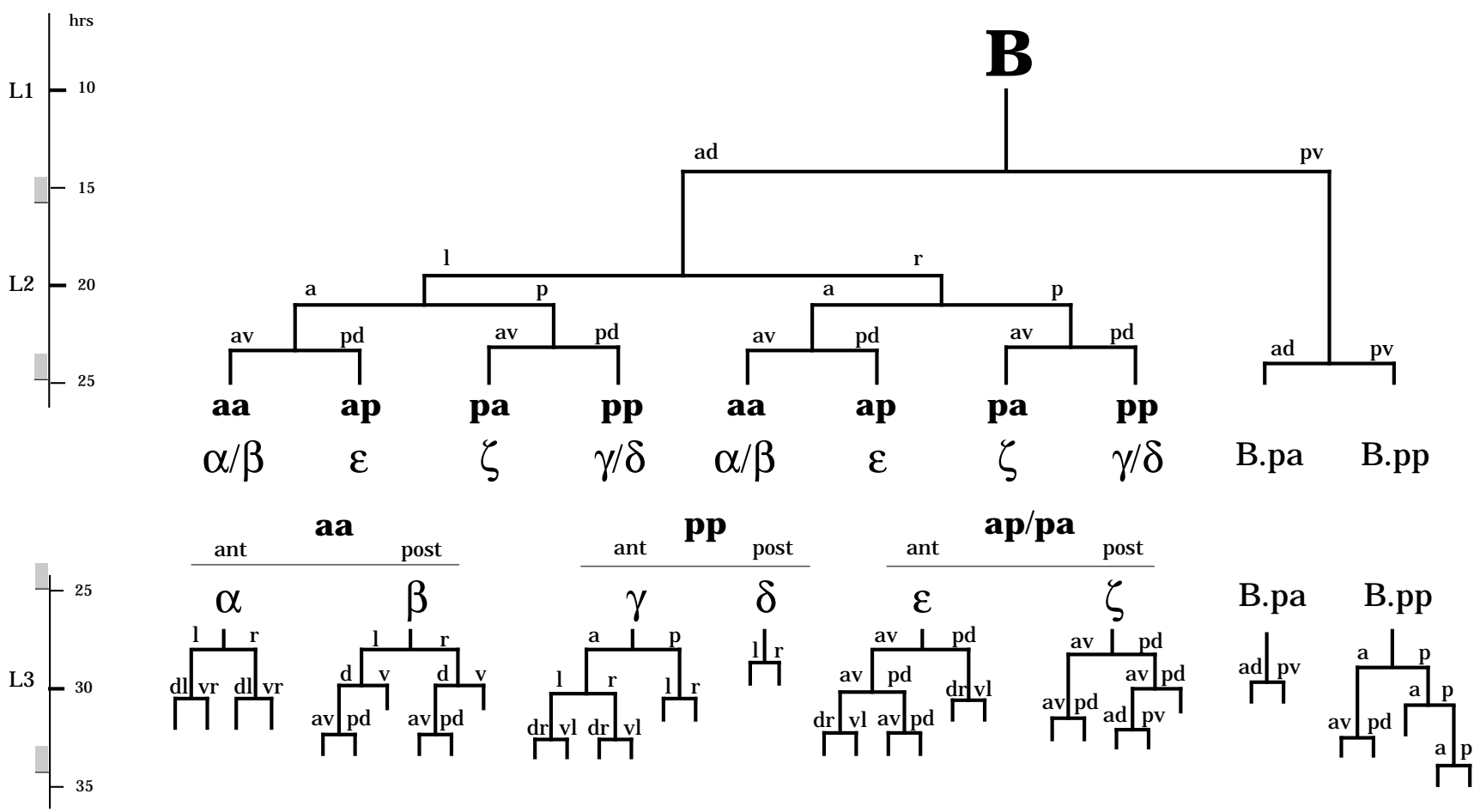

Fig. 2. The lineage of the C. elegans male B cell, after Sulston and Horvitz (1977) and Sulston et al. (1980). Vertical lines indicate a cell, horizontal lines indicate a cell division. Larval stage and approximate developmental time post-hatching are indicated in the left margin. Division axes are as indicated: a, anterior; $\mathrm{p}$, posterior; $\mathrm{d}$, dorsal; $\mathrm{v}$, ventral; 1 , left; $\mathrm{r}$, right.

unusual mutations. Also, we wanted to identify genotypes that may represent severe reduction of gene function for the male tail. The extent of the lineage defects can be variable both between and within given genotypes. This variability may be due to the alleles retaining partial gene activity.

Mutations used are described by Brenner (1974), Hodgkin et al. (1988) and as noted below.

Linkage Group (LG) II: clr-1(e1745). let-23(sy97), (sy278) and (n2020) (Aroian and Sternberg, 1991; H. M. C. and P. W. S., unpublished; S. Clark and R. Horvitz, unpublished). unc-4(e120).

LG III: lin-12(n137) and (n137n720) (Greenwald et al., 1983).

LG IV: unc-24(e138). mec-3(e1338). lin-3(n378), (n1058), (n1059) and (sy53) (Ferguson and Horvitz, 1985; Hill and Sternberg, 1992). lin-45(sy96) (Han et al., 1993). let-60(n2021), (sy95dn), (sy100dn), (n1046gf) and (sy103gf) (Beitel et al., 1990; Han and Sternberg, 1991; G. Jongeward, unpublished). dpy-20(e1282). unc-22(s7). unc31(e169). nT1[unc(n754) let] (=DnT1 balancer; Ferguson and Horvitz, 1985).

LG V: him-5(e1467) and (e1490) (Hodgkin et al., 1979).

LG X: sem-5(n1619) (Clark et al., 1992). lin-15(e1763), (n309) and (n377) (Ferguson and Horvitz, 1985).

Transgenic strains: PS1226 unc-31(e169); syEx21(hsp::lin-3). PS1238 unc-31(e169); syEx23(hsp::lin-3) (R. J. Hill, unpublished). These strains include multiple copies of a transgene with the EGFencoding domain of $\operatorname{lin}-3$ and a synthetic signal sequence under control of the $h s p 16$ promoter/enhancer (Stringham et al., 1992). The transgenes are maintained as extrachromosomal arrays. Although the transgenes produce only a portion of the $\operatorname{lin}-3$ gene, for simplicity we often refer to the product as LIN-3 (rather than the more accurate LIN3 EGF domain) as it represents lin-3 activity. Genomic unc-31 DNA was also included as a selectable marker.

Full genotypes of animals in Tables 1, 2 and 3 are as follows: him5(e1490) doubles were constructed for let-23(sy97), let-23(sy278), let-60(n2021), lin-45(sy96), let-60(n1046gf), let-60(sy103gf), lin15(e1763), lin-15(n309), lin-15(n377) and lin-12(n137). Data origi- nally summarized in Greenwald et al. (1983) (Tables 2 and 3) are from lin-12(n137); him-5(e1467) and lin-12(n137n720); him-5(e1467) animals. let-23(n2020) is let-23(n2020) unc-4(e120); him-5(e1490).

Other genotypes and construction:

lin-3(sy53)/lin-3(n1058): unc-24(e138) mec-3(e1338) lin-3(sy53) dpy-20(e1282) / lin-3(n1058). Construction: unc-24(e138) mec3(e1338) dpy-20(e1282)/+ males were crossed with lin3(n1058)/DnT1 hermaphrodites. Single non-Unc cross males (genotype: unc-24(e138) mec-3(e1338) dpy-20(e1282)/lin-3(n1058) or $+/$ lin-3(n1058)) were crossed with unc-24(e138) mec-3(e1338) lin3(sy53) dpy-20(e1282)/DnT1 hermaphrodites. non-Dpy non-Unc animals from crosses that yield Dpy Unc progeny (indicating paternal genotype of unc-24(e138) mec-3(e1338) dpy-20(e1282)/lin-3(n1058)) are the desired genotype.

lin-3(n378)/lin-3(n1059): lin-3(n378) / unc-24(e138) lin-3(n1059) dpy-20(e1282); him-5(e1490)/+. Construction: lin-3(n378); him5(e1490) males were crossed with lin-3(n1059)/DnT1 hermaphrodites. non-Unc male cross progeny are the desired genotype.

sem-5(n1619): clr-1(e1745)/+; sem-5(n1619). Construction: N2 males were crossed with $\operatorname{clr}-1($ e1745); sem-5(n1619). clr-1(e1745) suppresses the lethality associated with sem-5(n1619) and maternally rescues for lethality. However, the male tail defect is still observed.

let-60(dn): let-60(sy95)/dpy-20(e1282); him-5(e1490) or unc24(e138) let-60(sy100) dpy-20(e1282)/unc-22(s7); him-5(e1490).

Double mutant strains of let-23 or lin-45 and lin-15 were constructed according to standard methods (Ferguson et al., 1987). In addition to the two mutations, double mutant strains include him5(e1490). let-23(sy278); lin-15(e1763) is let-23(sy278) unc-4(e120); him-5(e1490); lin-15(e1763).

\section{Cell lineage and ablation}

Cell nuclei divisions in living animals were directly observed using Nomarski differential interference contrast optics as described by Sulston and Horvitz (1977). Nomenclature follows the standard of Sulston and Horvitz (1977), with modifications of Chamberlin and 
Sternberg (1993). All lineages were followed from the first divisions of the B.a(1/r)xx cells (early to mid L3 larval stage; $x$ represents both progeny of a division) through the L3 molt. Cell nuclei were destroyed by a laser microbeam as described by Avery and Horvitz (1987). F, $\mathrm{U}$ and Y.p were ablated at the stage when B had divided to produce two progeny (early L2), according to the procedures in Chamberlin and Sternberg (1993). Ablation of B.a progeny was during mid to late L2 stage, soon after the targeted cells were generated. Ablation of the 'B.a positional cue' for the aa cells in Table 3 represents the ablation of B.a(1/r)p, followed by the ablation of B.a(l/r)ap.

\section{Heat-shock induction of lin-3 transgenes}

Transgenic animals received heat shock at late L2 larval stage as follows. Individual animals were anaesthetized on pads of $5 \%$ noble agar in water containing $5 \mu \mathrm{M}$ sodium azide, staged and allowed to recover one hour on a standard $5 \mathrm{~cm}$ NGM agar Petri plate seeded with $E$. coli OP50. Plates were then sealed with parafilm and floated in a $33^{\circ} \mathrm{C}$ water bath for 90 minutes to induce the heat-shock response.

\section{RESULTS}

\section{The lin-3/let-23 signalling pathway mediates the $F / U$ signal during male spicule development}

We have examined the effects of increasing and decreasing the activity of the lin-3/let-23 pathway using chromosomal mutations and extrachromosomal transgenes. Taken together the results indicate that activation of the pathway is both necessary and sufficient to promote anterior fates, and it likely mediates the positional cue provided by $\mathrm{F}$ and $\mathrm{U}$.

\section{Mutations in some genes in the lin-3/let-23 pathway disrupt fates of anterior cells in the male $B$ lineage}

Some mutations in a subset of genes in the lin-3/let-23 signalling pathway result in an abnormal male spicule phenotype (see Fig. 1; also Aroian and Sternberg, 1991). These genes include lin-3, let-23, sem-5, let-60 and lin-45. In the B lineage, reduction-of-function mutations in any of these genes disrupt the lineages of the anterior cells (Table 1B,210). For instance, of seven lin-3(sy53)/lin3(n1058) animals followed, none had a normal $\alpha$ or a normal $\gamma$ lineage and only one had normal $\varepsilon$ lineages (Table $1 \mathrm{~B}, 2$ ). The phenotypes are similar to the abnormalities that result from ablation of both $\mathrm{F}$ and $\mathrm{U}$ in wildtype males (Table $1 \mathrm{~B}, 1)$. In some cases the anterior cells produce a lineage similar to their posterior neighbors. For instance, in all seven lin-3(sy53)/lin-3(n1058) animals both aa cells produced $\beta$ lineages.

Although the mutant phenotypes resemble the effects of $\mathrm{F} / \mathrm{U}$ ablation, the $\mathrm{F}$ and $\mathrm{U}$ cells appear normal in the mutants. The $\mathrm{F}$ and $\mathrm{U}$ lineages are normal in mutant animals (lineages normal in 2/2 lin-3(sy53)/lin3(n1058) and 2/2 lin-45(sy96) animals). In addition, the linker cell in the gonad dies as in wild-type animals (10/10 lin-3(sy53)/lin3(n1058) and 8/8 lin-45(sy96) animals). Since U.(1/r)p 'murder' the linker cell in intact animals (Sulston and White, 1980), these U progeny are still capable of at least one of their normal functions.
The pp cell lineage defect in lin-3 and lin-45 mutants is enhanced by ablation of $F$ and $U$ (Table 1B,11,12). For example, in $\mathrm{F}^{-} \mathrm{U}^{-}$lin-3(sy53)/lin-3(n1058) animals (Table $1 \mathrm{~B}, 11)$ both anterior and posterior $\mathbf{p p}$ cells produced $\delta$ lineages (posterior fate) in all seven animals. In contrast, although anterior pp lineages were disrupted, this pattern was observed in only one of seven lin-3(sy53)/lin-3(n1058) intact animals (Table 1B,2). However, ablation of $\mathrm{F}$ and $\mathrm{U}$ in mutant animals does not result in a consistent transformation of all anterior cells to posterior fate. In particular, although the lineages of anterior ap cells were disrupted, the ap cells did not produce $\zeta$ lineages.

\section{Ectopic expression of the ligand, LIN-3, disrupts the fates of posterior cells}

To test further the role of this signalling pathway in the $\mathrm{B}$ lineage, we used a transgenic construct that includes the EGFcoding domain of lin-3 under control of a broadly expressed heat-shock promoter (R. J. Hill, personal communication) that expresses in many tissues (Stringham et al., 1992). In the B lineage, heat-shock treatment of transgenic animals results in disruption of the lineage of the posterior cells (Table 1C,1,2). In the aa and ap/pa pairs, the posterior cells produce lineages normally associated with their anterior neighbors. For example, in five of six heat-shocked syEx21 animals followed, both aa cells produced $\alpha$ lineages and both ap and pa cells produced $\varepsilon$ lineages (Fig. 4).

In the pp pair, the posterior cells produce more anterior-like lineages. However, lineages of both anterior and posterior cells in this pair can be disrupted to produce up to eight progeny. Such abnormal lineages are observed following ablation of the ap/pa cells in wild-type (Chamberlin and Sternberg, 1993) as well as in the transgenic animals. Although abnormal, we believe these lineages may represent a transformation of all four pp daughters to the fate of the anterior daughter of $\gamma(\gamma . \mathrm{a}$; see Fig. 2). Specifically, cell ablation experiments suggested
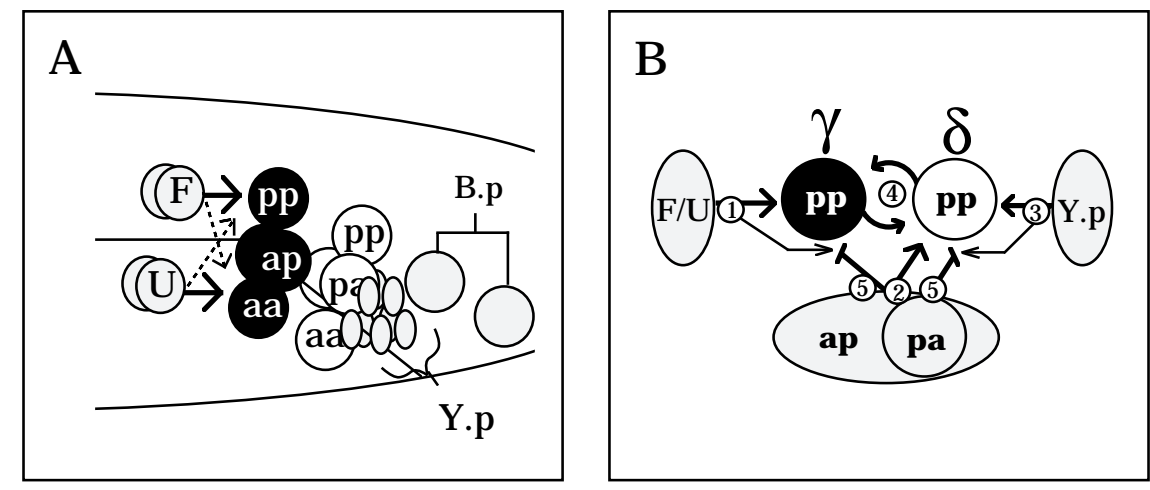

Fig. 3. (A) Diagram illustrating the approximate positions of the B, F, U and Y.p progeny in a mid-L3 larval stage male (anterior left, ventral down). Arrows indicate the signal from $\mathrm{F}$ and $\mathrm{U}$ that promotes the anterior fates in the $\mathbf{a a}$, ap/pa and pp pairs of $\mathrm{B} . \mathrm{a}$ progeny. (B) The signal from $F$ and $U$ (1) represents one of several cell interactions that specify fates in the B.a progeny, as illustrated in this model for the pp cells (after Chamberlin and Sternberg, 1993). Other positional cues (arrows) are provided by Y.p, or its progeny (3) and the other neighboring B.a progeny (2). In addition, the neighboring B.a progeny act to prevent the F/U and Y.p cues from acting on inappropriate cells (bars, 5). This interaction may be passive (Chamberlin and Sternberg, 1993). The two pp cells may also interact (4) (Greenwald et al., 1983; this work). There is no evidence that B.p provides any positional cue. Cells in black represent anterior fate (aa, $\alpha ; \mathbf{a p}, \varepsilon ; \mathbf{p p}, \gamma)$, white represent posterior fate (aa, $\beta ; \mathbf{p a}, \zeta ; \mathbf{p p}, \delta)$. 


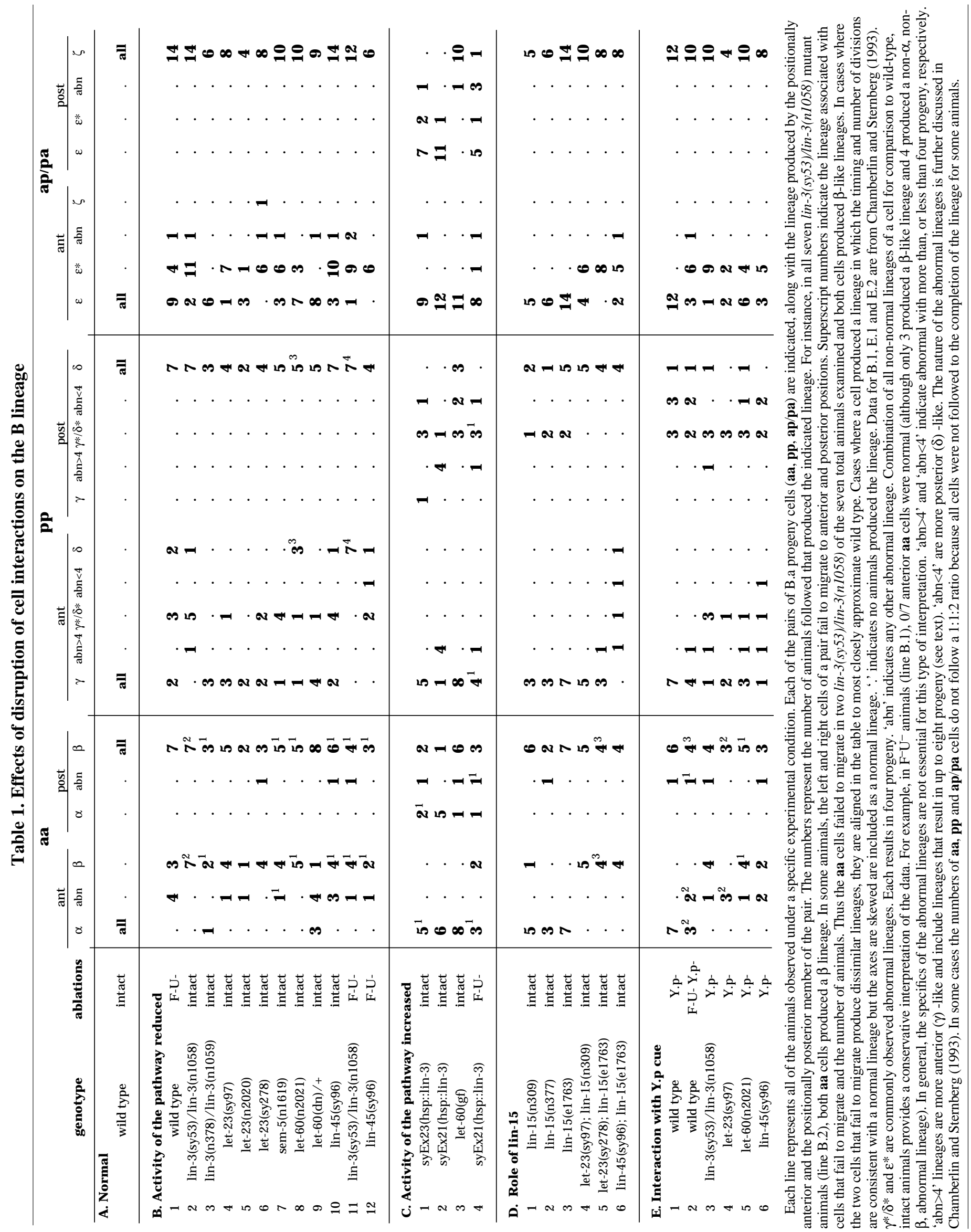


that patterning of $\gamma$ and $\delta$ fates may occur in two steps: first, the $\mathrm{F}$ and $\mathrm{U}$ signal promotes anterior versus posterior fate in the pp cells; second, it promotes $\gamma$.a versus $\gamma . p$ fate in the daughters of the anterior pp cell. According to this model, if the signal is not localized or 'modulated' both pp cells may adopt the anterior fate and both daughters may adopt the $\gamma . \mathrm{a}$ fate (Chamberlin and Sternberg, 1993). The observed result is up to eight progeny from each pp cell. The lineages observed in the transgenic animals that are presumably expressing the LIN-3 EGF domain ubiquitously (and hence the signal is not localized) are consistent with this model.

Ubiquitous LIN-3 is also sufficient to compensate for the absence of $F$ and $U$ (Table 1C,4), suggesting that activation of the lin-3/let-23 pathway is sufficient to promote anterior fates. Gain-of-function let-60 mutations that result in an activated protein also disrupt posterior fate, but to a lesser extent than ubiquitously expressed LIN-3 (Table 1C,3).

\section{Two activities that act antagonistically to lin-3/let-23 are integrated at functionally distinct steps in the pathway}

Mutations in lin-15 disrupt the fate of posterior pp cells

In the hermaphrodite vulva, reduction-of-function mutations in the lin-15 locus result in a phenotype opposite from reductionof-function mutations in the lin-3/let-23 genes required for vulval fates. In the male tail, mutations in lin-15 disrupt some posterior fates (Table 1D,1-3). However, even in lin-15 null mutants (e1763 represents a deletion of the lin-15 locus (Huang et al., 1994)), the lineage defect is only observed in some animals and only the lineage of the posterior pp cell is usually disrupted: it divides to produce up to four progeny instead of the normal two. Thus, in the B lineage mutations in lin-15 result in a phenotype opposite from those seen in lin-3/let-23 mutants. However, the effect is partial: spicule morphology in 31/63 (49\%) lin-15(n309), 23/55 (42\%) lin-15(n377) and 23/57 (40\%) lin-15(e1763) adult males is abnormal, consistent with the observation that the B lineage is abnormal in only 5/13 (38\%) lin-15 mutant animals followed. The effect is also primarily in the pp cell pair.

\section{lin-15 likely acts as a negative regulator of let-23 in the male $B$ lineage}

We followed the B lineage in a subset of double mutants to confirm the position of lin-15 in the lin-3/let-23 genetic pathway. Mutations in both let-23 and lin-45 block the requirement for functional lin-15 (Table 1D,4-6). Specifically, whereas in lin-15 mutants the lineage of the posterior pp cell can be disrupted, it is not disrupted in the let-23; lin-15 or lin-45; lin-15 double mutants. In addition, the lineage of the anterior pp cell can be disrupted as it is in let-23 or lin-45, but never lin-15, mutants. These results are consistent with lin-15 acting in the B lineage as it does in vulval development, where it negatively regulates let-23.

The positional cue from Y.p represents a signalling pathway distinct from lin-15

Y.p, or its progeny, produces a positional cue that promotes posterior fate, especially in the pp pair (Chamberlin and Sternberg, 1993). Ablation of Y.p disrupts the lineage of the posterior pp cell and the lineage defect is similar to that observed in lin-15 mutants (Table 1E,1). This suggested that Y.p may be the source of a signal mediated by lin-15. Ablation of F, U and Y.p together results in a disruption of both anterior and posterior pp cell lineages (Table 1E,2). Ablation of Y.p in lin-3, let-23, let-60 or lin-45 mutants resembles $\mathrm{F}^{-} \mathrm{U}^{-} \mathrm{Y} . \mathrm{p}^{-}$animals in that the fate of both anterior and posterior pp cells are commonly disrupted (Table 1E,3-6). In no case is a genetic mutation epistatic to the requirement for the presence of the Y.p cell. The similar results obtained for lin-3, let-23, let-60 and lin-45 mutants following Y.p ablation indicate that the positional information from Y.p does not act on the lin-3/let-23 signalling pathway upstream of lin-45 raf. Thus, lin-15 and the Y.p signal are functionally distinct.

\section{lin-12 can mediate a lateral interaction between the pp cells}

Reduction-of-function mutations in lin-12 (lin-12(0)) result in both pp cells producing $\gamma$-like lineages (anterior fate). In contrast, no B.a lineage defects are observed in animals bearing lin-12 gain-of-function mutations (lin-12(d)) (Greenwald et al., 1983). To better understand the role of lin-12 in pp fate specification, we have carried out cell ablation experiments in lin$12(d)$ mutants. Our results suggest that $\operatorname{lin}-12(d)$ mutations result in the opposite transformation from lin-12(0) mutations if $\mathrm{F}$ and $\mathrm{U}$ are ablated (Table 2). In lin-12(d) mutants both pp cells produce $\delta$ lineages when $\mathrm{F}$ and $\mathrm{U}$ are removed. However, since this result is at least similar to the effect observed when $\mathrm{F}$ and $\mathrm{U}$ are ablated in genotypically wild-type animals, the
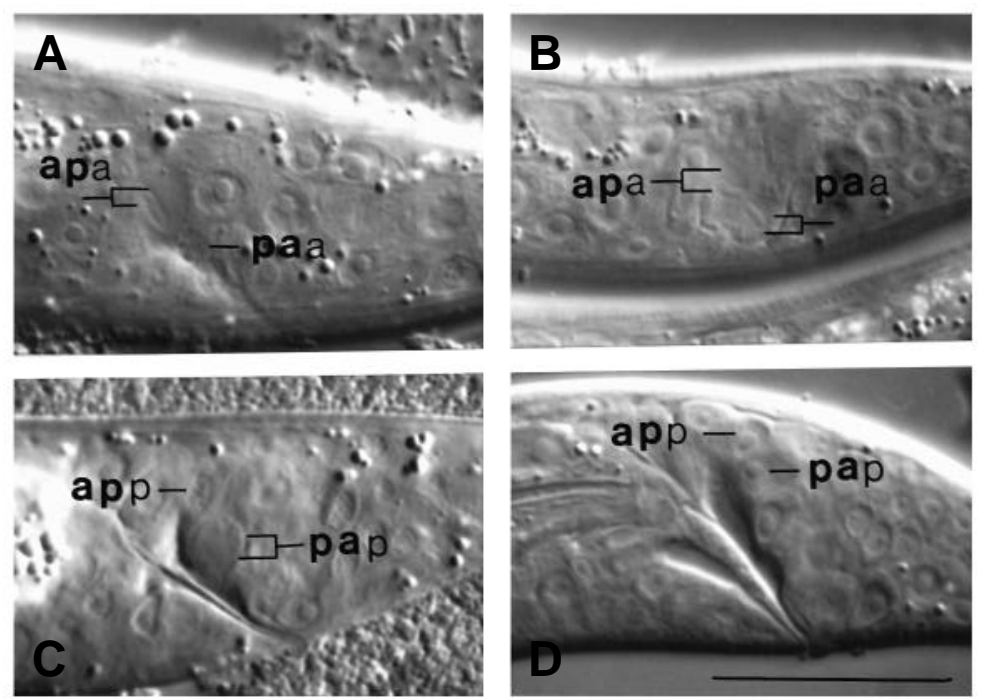

Fig. 4. Transformation of pa cells to $\varepsilon$ fate in animals with the generally expressed $h$ sp::lin-3 transgene. Nomarski photomicrographs compare wild type to heat shocked, transgenic animals carrying syEx21 (anterior left, ventral down). Differences between $\varepsilon$ and $\zeta$ lineages are apparent in the timing of division of the progeny of ap and pa. In wild-type animals $(A, C)$, ap cells produce $\varepsilon$ lineages and pa cells produce $\zeta$ lineages. In a normal $\varepsilon$ lineage, the anteroventral daughter (apa, A; metaphase plate is visible) divides prior to the posterodorsal daughter (app, C). In a normal $\zeta$ lineage, pap divides prior to paa. Ectopic LIN-3 can promote posterior cells to produce the lineages normally associated with their anterior neighbors. In such animals, the anteroventral daughters of both ap and pa (B; metaphase plates are visible) divide prior to the posterodorsal daughters (D) and both cells produce $\varepsilon$ lineages. Scale, $20 \mu \mathrm{m}$. 
Table 2. The role of lin-12 in the pp pair

\begin{tabular}{|c|c|c|c|c|c|c|c|c|c|}
\hline & \multirow[b]{3}{*}{$\mathrm{F} / \mathrm{U}$} & \multirow[b]{3}{*}{ lin-12 } & \multirow[b]{3}{*}{ Y.p } & \multicolumn{6}{|c|}{ pp } \\
\hline & & & & & nteric & & & steric & \\
\hline & & & & $\gamma$ & $\mathrm{abn}$ & $\delta$ & $\gamma$ & abn & $\delta$ \\
\hline a. & + & + & + & all & . & . & & & all \\
\hline b. & + & - & - & $3^{2}$ & & . & $2^{2}$ & 1 & 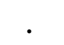 \\
\hline c. & + & d & + & 3 & 1 & & . & & 4 \\
\hline d. & - & + & + & 2 & 3 & 2 & . & . & 7 \\
\hline e. & - & d & + & & & $3^{1}$ & . & & $\mathbf{3}^{1}$ \\
\hline f. & - & + & - & 4 & 1 & & . & 4 & 1 \\
\hline g. & - & d & - & & . & $5^{3}$ & . & & $5^{3}$ \\
\hline
\end{tabular}

In lin-12(0) animals, both pp cells adopt $\gamma$ fate (b). In lin-12(d) animals, the pp cells are normal. Ablation of the positional cues from F/U (e) uncovers the defect in lin-12(d) animals and both pp cells adopt $\delta$ fate. The contrast between lin-12(d) and wild type is best seen when F, U and Y.p are all removed (compare $\mathrm{f}$ to $\mathrm{g})$. Each line indicates the presence of the F/U positional cue, lin-12 genotype (+, wild type; -, loss-of-function (lin-12(0)); d, gain-of-function (lin-12(d)) and

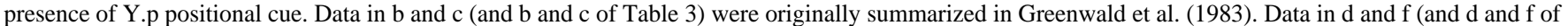
Table 3) are from Chamberlin and Sternberg (1993). In lin-12(0) animals (b), the Y cell adopts a neuronal fate and thus the Y.p cue is absent. In lin-12(d) animals $(\mathrm{c}, \mathrm{e}, \mathrm{g})$ there are two Y.p-like cells. Both cells were ablated in the animals of line g. Notation is as in Table 1.

Table 3. The role of lin-12 in the aa pair

\begin{tabular}{|c|c|c|c|c|c|c|c|c|c|c|}
\hline & \multirow[b]{2}{*}{$\mathrm{F} / \mathrm{U}$} & \multirow[b]{2}{*}{ lin-12 } & \multirow[b]{2}{*}{ Y.p } & \multirow[b]{2}{*}{ B.a } & \multicolumn{2}{|c|}{ anterior } & \multirow[b]{2}{*}{$\beta$} & \multicolumn{3}{|c|}{ posterior } \\
\hline & & & & & $\alpha$ & abn & & $\alpha$ & abn & $\beta$ \\
\hline a. & + & + & + & + & all & & . & . & . & all \\
\hline b. & + & - & - & + & 2 & & . & . & . & 2 \\
\hline c. & + & d & + & + & 3 & & . & . & $\cdot 1$ & 3 \\
\hline d. & - & + & - & + & $3^{2}$ & $2^{2}$ & . & . & $\mathbf{1}^{1}$ & $4^{3}$ \\
\hline e. & - & $d$ & - & + & $2^{2}$ & $2^{2}$ & . & . & $2^{2}$ & $2^{2}$ \\
\hline f. & - & + & - & - & $6^{5}$ & & & $\cdot$ & $3^{3}$ & $3^{2}$ \\
\hline g. & - & $d$ & - & - & 1 & 1 & $\mathbf{1}^{1}$ & . & 1 & $2^{2}$ \\
\hline
\end{tabular}

aa lineages are normal in both lin-12(0) and lin-12(d) mutants. Removal of F/U, Y.p and B.a positional cues in wild-type animals uncovers a possible lateral interaction between aa cells (Chamberlin and Sternberg, 1993; f). Removal of F/U, Y.p and B.a positional cues in lin-12(d) mutants indicates that lin-12 does not appear to play an essential role in the specification of aa fates even in the absence of these positional cues. Notation is as in Tables 1 and 2.

contrast between lin-12(d) and wild type is best seen when F, $\mathrm{U}$ and Y.p are removed. In wild-type animals, this ablation results in $\gamma$ and $\gamma^{*} / \delta^{*}$ lineages from both pp cells, whereas in lin-12(d) animals both pp cells produce $\delta$ lineages (posterior fate; compare Table $2 \mathrm{f}, \mathrm{g}$ ). Thus, the presence of $\mathrm{F}$ and $\mathrm{U}$ can override the effect of the lin-12(d) mutation. An additional defect in lin-12(0) animals is that the presumptive $\mathrm{Y}$ cell is transformed to a neuronal fate similar to its lineal homolog DA9 (Greenwald, et al., 1983). Since lin-12(0) animals are also missing the positional cue from Y.p, we cannot yet establish if the presence of Y.p is likewise sufficient to compensate for the absence of lin-12 activity.

Ablation experiments in wild-type males suggested that the aa cells may also interact with each other (Chamberlin and Sternberg, 1993). However, ablation experiments in lin-12(d) mutants carried out for the aa pair failed to provide evidence of an essential role for lin-12 (Table 3).

\section{DISCUSSION}

\section{The genes in the lin-3/let-23 signalling pathway mediate the F/U signal defined by cell ablation}

Cell ablation experiments suggest that four distinct cell interactions are essential for normal anterior/posterior patterning of fates for four pairs of cells in the C. elegans male B lineage (Chamberlin and Sternberg, 1993). The male-specific blast cells $\mathrm{F}$ and $\mathrm{U}$ mediate one of these signals that is necessary for normal anterior fates. The lin-3/let-23 pathway is both necessary and sufficient to promote anterior fates, and thus likely mediates the $\mathrm{F} / \mathrm{U}$ signal.

The lineage defect in lin-3/let-23 mutants with $\mathrm{F}$ and $\mathrm{U}$ ablated is more severe than either mutant or ablation alone. This result suggests that not only might the analyzed mutations not represent null mutations for male tail function, but also that all signalling activity may not be eliminated from animals in which $\mathrm{F}$ and $\mathrm{U}$ have been ablated. Such residual activity may come from the debris of the ablated cells, or may come from other, unidentified sources. However, if such other sources exist they are not sufficient to promote the normal anterior fates in the absence of $\mathrm{F}$ and $\mathrm{U}$, and likely do not play a significant role in normal development.

\section{The role of lin-3/let-23 in fate specification}

What specific role in fate specification does the lin-3/let-23 pathway play in $C$. elegans spicule development? Our results suggest that the same genes mediate the F/U signal for all four pairs of B.a progeny. Since mutations in a single gene of the lin-3/let-23 pathway can result in all of the defects observed in animals with $\mathrm{F}$ and $\mathrm{U}$ ablated, we can exclude the possibility 


\section{pp cells}

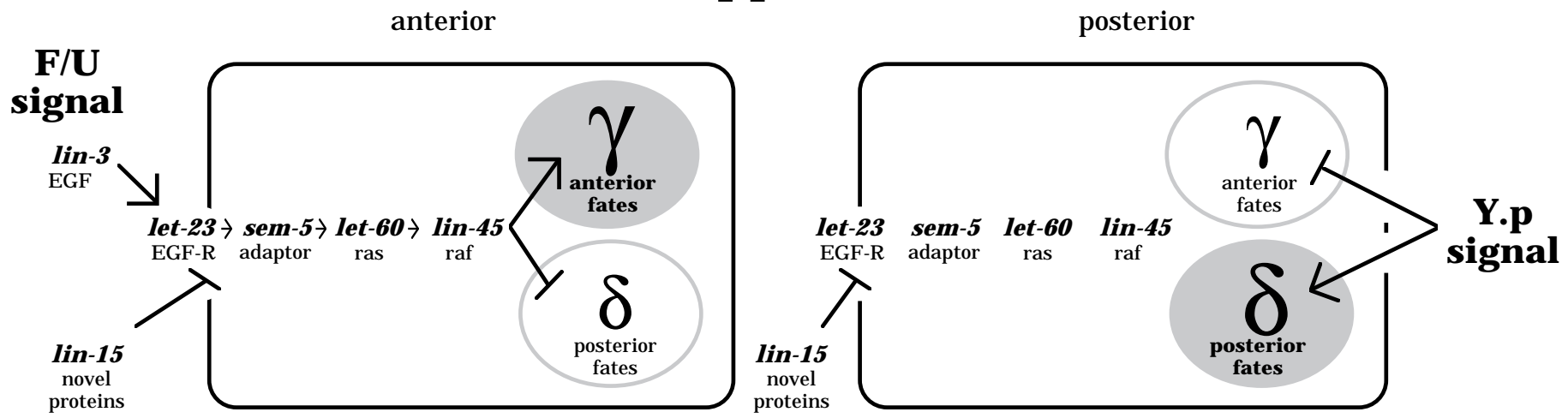

Fig. 5. Site of action of lin-15 and the Y.p cue relative to the lin-3/let-23 pathway. lin-15 likely acts antagonistically and in parallel to lin-3 as a negative regulator of let-23 activity to specify fates in the B lineage. The Y.p signal acts on the pathway downstream of lin-45 and may represent an independent signalling pathway that acts in parallel to, or possibly after, the lin-3/let-23 pathway. The gene order in the pathway is based on the epistasis established for these genes in hermaphrodite vulval induction (reviewed by Sternberg, 1993). It is not known if the genes are expressed in the indicated cells.

that $\mathrm{F}$ and $\mathrm{U}$ produce three different signals, with one for the aa pair, one for the pp pair and one for the ap/pa pairs. Nevertheless, the cellular response is distinct for each pair. In response to generally expressed LIN-3, the pp cells produce more progeny than normal while aa cells produce fewer progeny. LIN-3 also does not appear to induce a specific differentiated cell type (Chamberlin and Sternberg, 1993), since neuronal and epidermal progeny arise from both anterior and posterior blast cells (Sulston et al., 1980). These differences among the three pairs suggest that the lin-3/let-23 pathway promotes a particular choice among possible responses. The final outcome depends upon functional differences among the responding cells.

\section{The role of lin-15 in the B lineage}

During $C$. elegans vulval development, loss-of-function, molecular null mutations in lin-15 result in all VPCs adopting vulval fates (Huang et al., 1994). The defect in the B lineage, in contrast, is less extreme and less than $50 \%$ penetrant. Both vulval development and spicule development, however, have a necessary requirement for the positive acting genes of the lin$3 /$ et-23 pathway. One possible reason for this difference in the requirement for lin-15 is that development of the B cell includes additional specification mechanisms, such as positional cues from Y.p and the other B.a progeny, that also act antagonistically to the lin-3/let-23 pathway. We believe that although the function of lin-15 may be similar in both developmental processes, its role is diminished in the B lineage as it is partially redundant with other activities.

\section{The role of lin-12 in the B lineage}

Our results support the findings of Greenwald et al. (1983) and suggest that lin-12 may mediate a lateral interaction between the two pp cells. However, lin-12 is not sufficient for $\delta$ fate, as the presence of the $\mathrm{F}$ and $\mathrm{U}$ cells can promote $\gamma$ fate in lin12(d) mutants. This result is similar to the relationship between lin-12(d) mutations and the AC signal in vulval development. Normally all VPCs adopt $2^{\circ}$ vulval fate in lin-12(d) mutants. However, these animals also lack an AC due to an earlier function of lin-12. In rare lin-12(d) animals with an AC, the
AC signal can override the $\operatorname{lin}-12(d)$ defect and promote $1^{\circ}$ fate in the most proximal VPC (Sternberg and Horvitz, 1989). In the B lineage, lin-12 may also not be necessary for $\delta$ fate, as we have not tested if a lin-12(0) animal with a normal Y cell can produce a normal pattern of pp cell fates. Thus, lin-12 functions in this cell pair, but is at least partially redundant with other positional cues.

\section{Integration of multiple signals}

Two activities that act antagonistically to the lin-3/let-23 pathway are required for normal fate specification in the dorsal pp pair (Fig. 5). If lin-15 acts in the B lineage as it does in vulval development and is required in cells other than the responding cells (Herman and Hedgecock, 1990), then both lin-15 and the Y.p positional cue represent extracellular cues that must be integrated by the responding cells. We have characterized the probable relationship of both these activities relative to the lin-3/let-23 pathway. If the function of a gene (like lin-15) or a signal (like the Y.p cue) is to negatively regulate the activity of the receptor let-23, mutations in the receptor or any downstream gene such as lin-45 should render the pathway insensitive to the removal of the regulator. Consequently, the lineage defect observed in let-23 or lin-45 mutants with the activity removed should be the same as when the activity is intact. This is the case with lin-15. In particular let-23; lin-15 and lin-45; lin-15 double mutants resemble the single let-23 and lin-45 mutants, with the posterior pp cell always producing a normal $\delta$ lineage. However, ablation of Y.p in let-23, let-60 or lin-45 mutants results in abnormal lineages similar to $\mathrm{F}^{-} \mathrm{U}^{-} \mathrm{Y} . \mathrm{p}^{-}$or lin-3 Y.p $\mathrm{p}^{-}$animals. In all cases, the posterior pp cell produces abnormal lineages rather than the normal $\delta$ lineage. Therefore, the Y.p cue does not act by negatively regulating the lin-3/let-23 pathway upstream of lin-45 raf. Furthermore, the anterior pp cell produces abnormal lineages as in the single mutants rather than the normal $\gamma$ lineage. Thus, disruption of the Y.p signal is not strictly epistatic to disruption of the $\mathrm{F} / \mathrm{U}$ signal. We propose that the Y.p cue represents a distinct, parallel signalling pathway that acts at the same time or later than the lin-3/let-23 pathway.

The positional cues from F/U and Y.p represent two of the 
four active cell interactions identified by cell ablation experiments (see Fig. 3B). F and U provide an anterior positional cue (labeled 1 in Fig. 3) that is mediated by the lin-3/let-23 signalling pathway. Our results and the results of Greenwald et al. (1983) suggest that, at least in the pp pair, the lateral interaction (4 in Fig. 3) is mediated by the gene lin-12. Our results indicate that lin-15 does not mediate the Y.p cue (3 in Fig. 3). However, our data do not rule out the possibility that lin-15 plays a role in mediating the positional cue from the other B.a progeny (2 in Fig. 3).

We have found that, in the $C$. elegans male B lineage, the fate of the pp cells results from coordinate integration of multiple signals, including three defined genetically by the lin3/let-23 pathway, lin-15 and lin-12 genes initially identified for their role in hermaphrodite vulval development. Not only do the pp cells respond to the same signalling pathways as do the hermaphrodite VPCs, but the integration of these signals appears to be similar: lin-15 acts as a negative regulator of let23 and the signal mediated by the lin-3/let-23 pathway can override the effect of a lin-12(d) mutation. Yet the cellular responses, such as cell division pattern and the differentiated fates of progeny that result in response to these signals are very different in male spicule development compared to hermaphrodite vulval development. An exciting possibility is that, in insect and vertebrate development, as well as in nematode development, not only are signalling pathways conserved, but so is the coordination of multiple signal integration.

We thank T. R. Clandinin, A. Golden, R. J. Hill, L. S. Huang, G. D. Jongeward, W. Katz, P. Kayne, H. D. Lipshitz, J. Liu, A. Newman, R. Palmer and R. Sommer for comments on the manuscript, and G. Medina and Y. Hajdu-Cronin for technical assistance. We also thank R. J. Hill for providing syEx21 and syEx23 and S. Clark for providing let-23(n2020) prior to publication. Some of the strains used in this study were provided by the Caenorhabditis Genetics Center, which is funded by the NIH National Center for Research Resources (NCRR). This research was supported by the Howard Hughes Medical Institute and an NSF Presidential Young Investigator Award to P. W. S.; H. M. C. was an NSF predoctoral fellow; P. W. S. is an investigator of the HHMI.

\section{REFERENCES}

Aroian, R. V., Koga, M., Mendel, J. E., Ohshima, Y. and Sternberg, P. W. (1990). The let-23 gene necessary for Caenorhabditis elegans vulval induction encodes a tyrosine kinase of the EGF receptor subfamily. Nature 348, 693-699.

Aroian, R. V. and Sternberg, P. W. (1991). Multiple functions of let-23, a C. elegans receptor tyrosine kinase gene required for vulval induction. Genetics 128, 251-267.

Avery, L. and Horvitz, H. R. (1987). A cell that dies during wild-type $C$. elegans development can function as a neuron in a ced-3 mutant. Cell $\mathbf{5 1}$, 1071-1078.

Beitel, G., Clark, S. and Horvitz, H. R. (1990). The Caenorhabditis elegans ras gene let-60 acts as a switch in the pathway of vulval induction. Nature 348, 503-509.

Brenner, S. (1974). The genetics of Caenorhabditis elegans. Genetics 77, 7194

Chamberlin, H. M. and Sternberg, P. W. (1993). Multiple cell interactions are required for fate specification during male spicule development in Caenorhabditis elegans. Development 118, 297-323.

Clark, S. G., Stern, M. J. and Horvitz, H. R. (1992). C. elegans cellsignalling gene sem-5 encodes a protein with $\mathrm{SH} 2$ and $\mathrm{SH} 3$ domains. Nature 356, 340-344.

Clark, S. G., Lu X. and Horvitz, H. R. (1994). The C. elegans locus lin-15, a negative regulator of a tyrosine kinase signaling pathway, encodes two different proteins. Genetics (in press).

Davidson, E. H. (1993). Later embryogenesis: regulatory circuitry in morphogenetic fields. Development 118, 665-690.

Ferguson, E. and Horvitz, H. R. (1985). Identification and characterization of 22 genes that affect the vulval cell lineages of Caenorhabditis elegans. Genetics 110, 17-72.

Ferguson, E. L., Sternberg, P. W. and Horvitz, H. R. (1987). A genetic pathway for the specification of the vulval cell lineages of Caenorhabditis elegans. Nature 326, 259-267.

Ferguson, E. and Horvitz, H. R. (1989). The multivulva phenotype of certain C. elegans mutants results from defects in two functionally-redundant pathways. Genetics 123, 109-121.

Greenwald, I. S., Sternberg, P. W. and Horvitz, H. R. (1983). The lin-12 locus specifies cell fates in Caenorhabditis elegans. Cell 34, 435-444.

Han, M., Aroian, R. and Sternberg, P. W. (1990). The let-60 locus controls the switch between vulval and non-vulval cell types in C. elegans. Genetics 126, 899-913.

Han, M., Golden, A., Han, Y. and Sternberg, P. W. (1993). C. elegans lin-45 raf gene participates in let-60 ras stimulated vulval differentiation. Nature 363, 133-140.

Han, M. and Sternberg, P. W. (1990). let-60, a gene that specifies cell fates during C. elegans vulval induction, encodes a ras protein. Cell 63, 921931.

Han, M. and Sternberg, P. W. (1991). Analysis of dominant negative mutations of the Caenorhabditis elegans let-60 ras gene. Genes Dev. 5 , 2188-2198.

Herman, R. K. and Hedgecock, E. M. (1990). The size of the C. elegans vulval primordium is limited by lin-15 expression in surrounding hypodermis. Nature 348, 169-171.

Hill, R. J. and Sternberg, P. W. (1992). The lin-3 gene encodes an inductive signal for vulval development in C. elegans. Nature 358, 470-476.

Hodgkin, J., Edgley, M., Riddle, D. L. and Albertson, D. G. (1988) Appendix 4, Genetics In The Nematode Caenorhabditis elegans (ed. W. B. Wood), pp. 491-584. Cold Spring Harbor, NY: Cold Spring Harbor Laboratory.

Hodgkin, J., Horvitz, H. R. and Brenner, S. (1979). Nondisjunction mutants of the nematode Caenorhabditis elegans. Genetics 91, 67-94.

Huang, L. S., Tzou, P. and Sternberg, P. W. (1994). The lin-15 locus encodes two negative regulators of $C$. elegans vulval development. Molec. Biol. Cell 5, 395-412.

Kimble, J. (1981). Lineage alterations after ablation of cells in the somatic gonad of Caenorhabditis elegans. Dev. Biol. 87, 286-300.

Kimelman, D., Christian, J. L. and Moon, R. T. (1992). Synergistic principles of development: overlapping patterning systems in Xenopus mesoderm induction. Development 116, 1-9.

Seydoux, G. and Greenwald, I. (1989). Cell autonomy of lin-12 function in a cell fate decision in C. elegans. Cell 57, 1237-1245.

Sternberg, P. W. (1988). Lateral inhibition during vulval induction in Caenorhabditis elegans. Nature 335, 551-554.

Sternberg, P. W. (1993). Intercellular signaling and signal transduction in $C$. elegans. Ann. Rev. Genet. 27, 497-521.

Sternberg, P. W. and Horvitz, H. R. (1989). The combined action of two intercellular signalling pathways specifies three cell fates during vulval induction in C. elegans. Cell 58, 679-693.

Stringham, E. G., Dixon, D. K., Jones, D. and Candido, E. P. M. (1992). Temporal and spatial expression patterns of the small heat shock (hsp16) genes in transgenic Caenorhabditis elegans. Mol. Biol. Cell 3, 221-233.

Sulston, J. and Horvitz, H. R. (1977). Postembryonic cell lineages of the nematode Caenorhabditis elegans. Dev. Biol. 56, 110-156.

Sulston, J. E., Albertson, D. G. and Thomson, J. N. (1980). The Caenorhabditis elegans male: Postembryonic development of nongonadal structures. Dev. Biol. 78, 542-576.

Sulston, J. E. and Hodgkin, J. (1988). Methods In The Nematode Caenorhabditis elegans (ed. W. Wood), pp. 587-606. Cold Spring Harbor, New York: Cold Spring Harbor Laboratory.

Sulston, J. E. and White, J. G. (1980). Regulation and cell autonomy during postembryonic development of Caenorhabditis elegans. Dev. Biol. 78, $577-$ 597.

Yochem, J., Weston, K. and Greenwald, I. (1988). The Caenorhabditis elegans lin-12 gene encodes a transmembrane protein with overall similarity to Drosophila Notch. Nature 335, 547-550.

(Accepted 29 June 1994) 